\title{
Tunable Multi Wavelength by Pulse Signal Modulation in Laser Pumping of EDFA
}

\author{
Poramate Chunpang1, Seckson Sukhasena2* \\ ${ }^{1}$ Department of Physics, Faculty of Science, Mahasarakham University, Mahasarakham, Thailand \\ ${ }^{2}$ The Institute for Fundamental Study, Naresuan University, Phitsanulok, Thailand \\ Email: poramate.p@msu.ac.th, ${ }^{*}$ secksons@nu.ac.th
}

Received 5 March 2015; accepted 30 March 2015; published 31 March 2015

Copyright (C) 2015 by authors and Scientific Research Publishing Inc.

This work is licensed under the Creative Commons Attribution International License (CC BY).

http://creativecommons.org/licenses/by/4.0/

(c) (i) Open Access

\begin{abstract}
This report is aimed to present the result of experimental setup of the erbium-doped fiber amplifier (EDFA) with modulated pulse signal by laser pumping at wavelength $980 \mathrm{~nm}$. The amplified spontaneous emission ( $A S E$ ) from EDFA has multi wavelength and the spacing of wavelength can be controlled by controlling the pulse width of laser pumping. The result in this experiment shows that the feasibility of using EDFA system can generate the multi wavelength of all of $C$-Band spectrum. The pulse signal, for modulated laser pumping, is observed ranging from 10 to $100 \mathrm{~Hz}$ and the wavelength spacing can be tuned from $14.7 \mathrm{~nm}$ to $14.9 \mathrm{~nm}$.
\end{abstract}

Keywords

Erbium-Doped Fiber Amplifier (EDFA), WDM, Multi Wavelength Laser

\section{Introduction}

Erbium-doped fiber amplifier (EDFA) is a widely applied component, e.g., for wavelength division multiplexing $(W D M)$ in modern optical communication systems, optical fiber sensing system, optical device testing system and optical instrumentation [1] [2]. In addition, the application of EDFA is a main device for generation of multi wavelength laser [3] [4]. The EDFA has gained spectrum operation range from $1525 \mathrm{~nm}$ to $1565 \mathrm{~nm}$ in the range of $C$-Band [5]. The EDFA is used successfully due to a high gain, low insertion loss, high output power and polarization-independent gain. There are many techniques of multi wavelength generation such as Fabry-Perot etalon inside cavity [6], fiber bragg grating [7], Sagnac interferometer [8], ring resonator [9], high nonlinear fiber [10], semi-conductor optical amplifier [11], $\mathrm{LiNbO}_{3}$ [12]. Most of these techniques are of high cost and require many equipments.

${ }^{*}$ Corresponding author.

How to cite this paper: Chunpang, P. and Sukhasena, S. (2015) Tunable Multi Wavelength by Pulse Signal Modulation in Laser Pumping of EDFA. Journal of Modern Physics, 6, 521-525. http://dx.doi.org/10.4236/jmp.2015.65056 
In this report, we carried out a novel method for experimental demonstration tunable multi wavelength and frequency spacing by modulate pulse signal into laser pumping. The purpose of this experiment is for a simpler setup for generation a multi wavelength laser by using EDFA.

\section{Experiments and Results}

The experimental setup is illustrated in Figure 1. The multi wavelength are formed by the wavelength division multiplexed (WDM) with 980/1550 multiplexing for the coupling light pumping source into the EDFA and for using in the future. The $L D$ driver at the input port, with $980 \mathrm{~nm}$, is connected to a pumping laser and the output port is connected to the optical isolator for improving the noise figure performance. A 20 meters EDFA length is connected between the optical isolators and the optical spectrum analyzer (OSA). The $L D$ driver is driving the current of laser diode pumping. And the modulator (Mod.) is modulated pulse signal by the pulse generator to control the current in the laser diode with the pulse frequency varying from $10 \mathrm{~Hz}$ to $100 \mathrm{~Hz}$ by increasing a step by step of $5 \mathrm{~Hz}$ frequency. The maximum power of the laser pumping is $50 \mathrm{~mW}$ while the pulse generator is setup for duty cycle at $50 \%$.

In this proposition, we will discuss and analyze the result of the EDFA with modulated pulse signal in the laser diode pumping. Figure 2 illustrates the results of an experimental setup of the proposing tunable multi wavelength of all of the $C$-Band Erbium-doped fiber amplifier with modulated laser pumping.

The resultant of the output port is showing the spectrum by OSA as in Figure 2. The output spectrum of the multi wavelength laser with spanning of wavelength $100 \mathrm{~nm}$. The resolution bandwidth of the optical spectrum analyzer is $0.06 \mathrm{~nm}$. The wavelength line spacing with modulated pumping laser in Figures 2(a)-(c) are 14.70 $\mathrm{nm}, \sim 2.94 \mathrm{~nm}$ and $\sim 1.49 \mathrm{~nm}$ respectively. From this experiment we describe the multi wavelength laser source with control spectral range by control frequency for modulation pumping laser. The peak power of multi wavelengths generate output are equal line spectral of amplified spontaneous emission (ASE) spectrum with continuous wave (CW) pumping laser at $50 \mathrm{~mW}$. The result of output spectrum with modulation pulse signal into the pumping laser is the same as by using CW pumping when the pulse signal is increasing too high frequency.

Figure 3 shows the result of the wavelength spacing with modulated pulse signal into the laser pumping at 10 $\mathrm{Hz}$ to $100 \mathrm{~Hz}$ by increasing step by step of the frequency by $5 \mathrm{~Hz}$. The experimental result, at $100 \mathrm{~Hz}$, have multi wavelength laser with multiple wavelength up to 67 wavelengths at all of $C$-Band. The spectral width is decreasing in exponential trend because of the nonlinear optical effectiveness and transient response of EDFA.

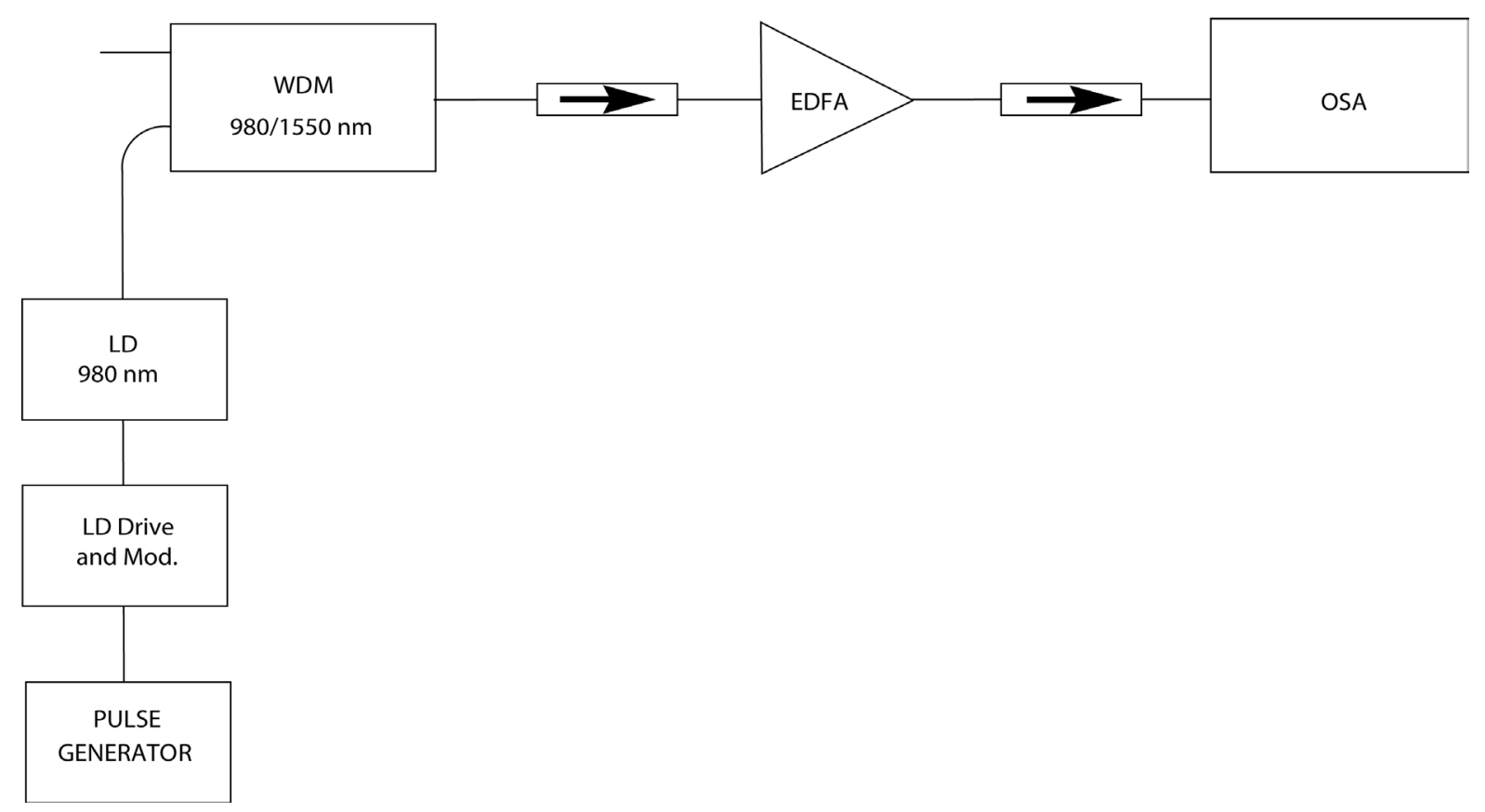

Figure 1. The experimental setup of an investigation of multi wavelength generating in EDFA by modulate pumping laser scheme. 

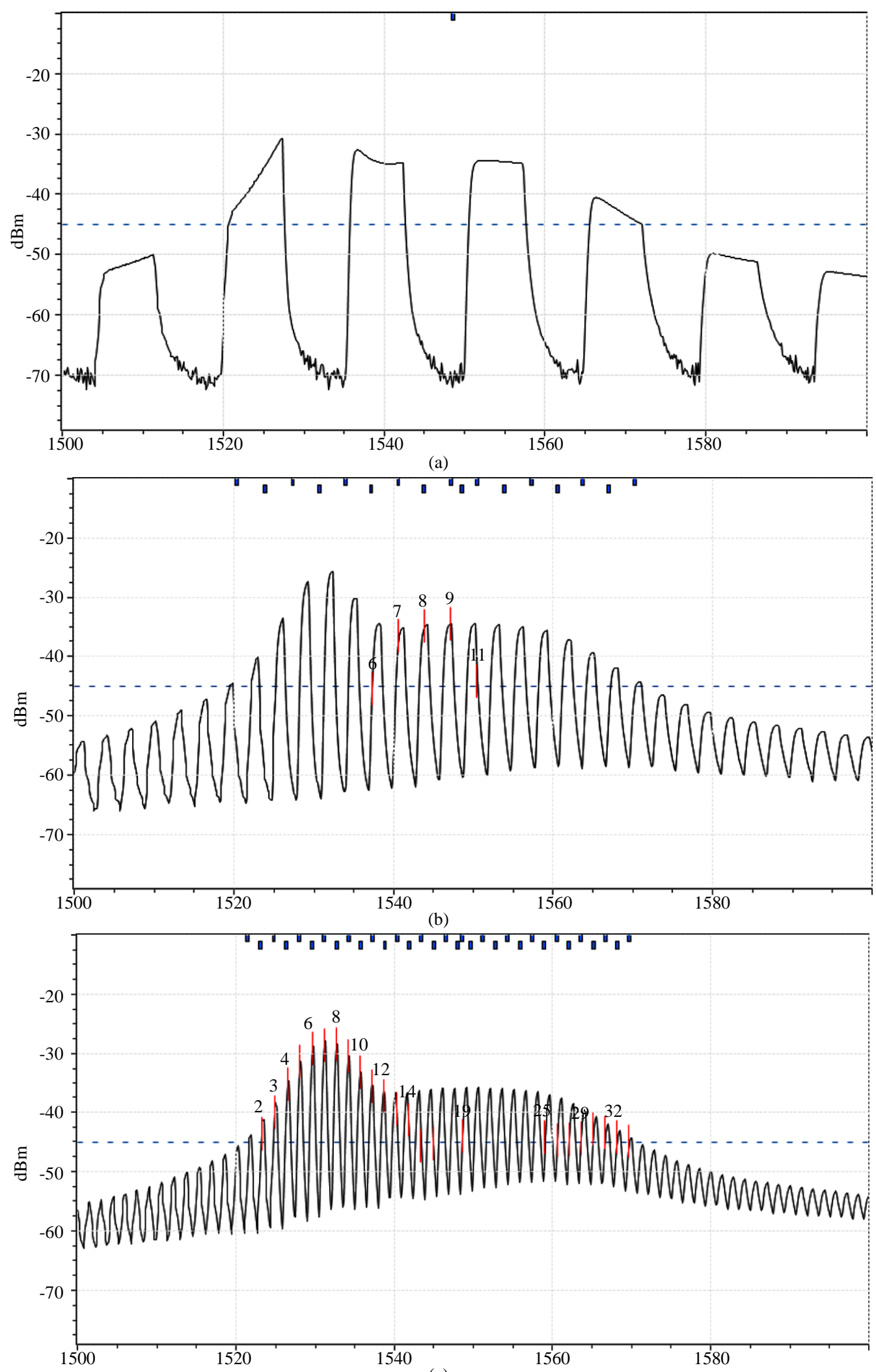

(c)

Figure 2. The spectrum of EDFA with power pumping laser $50 \mathrm{~mW}$ and modulated with $10 \mathrm{~Hz}, 50 \mathrm{~Hz}$ and $100 \mathrm{~Hz}$ are shown in (a), (b) and (c) respectively. 


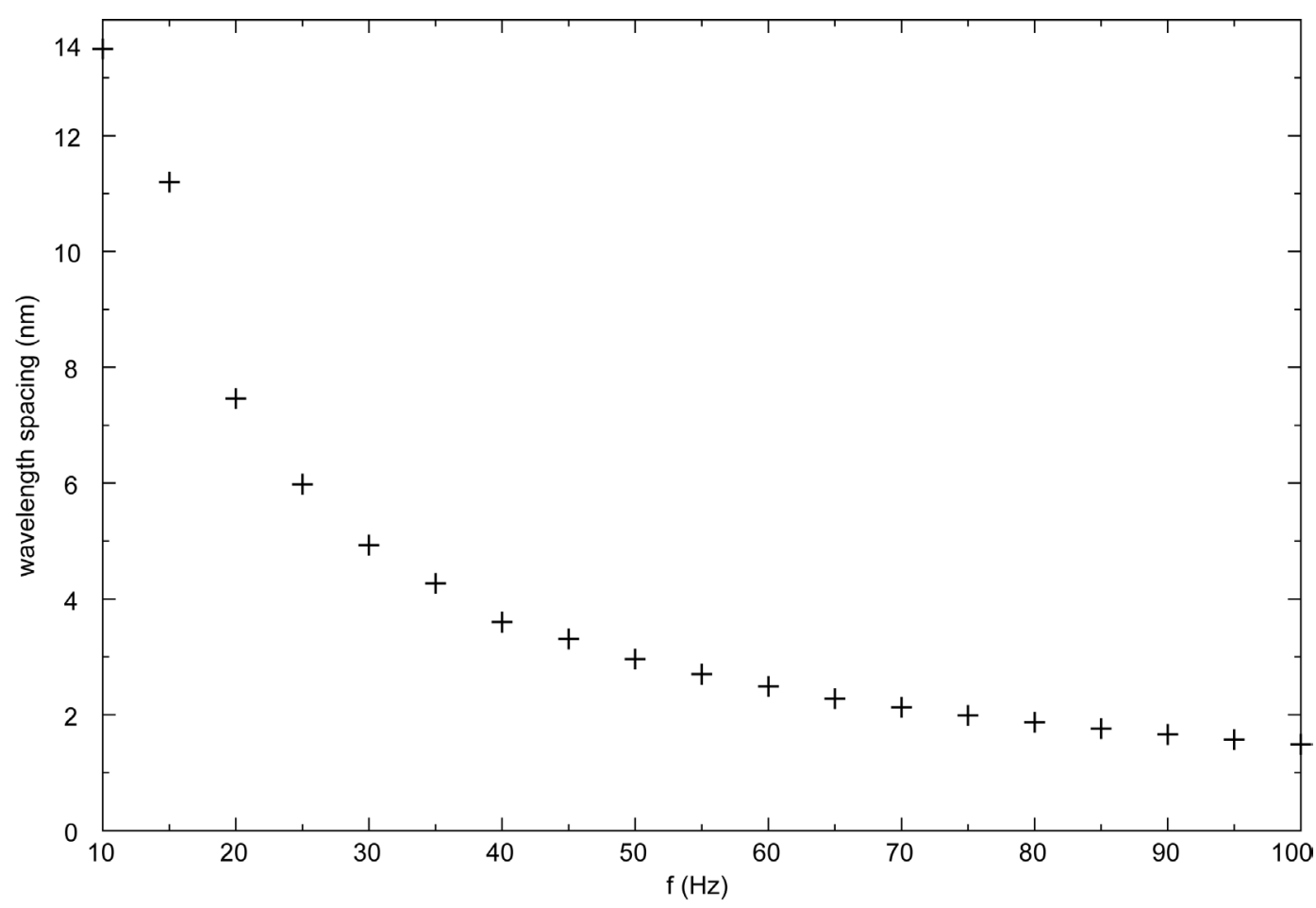

Figure 3. The experimental result showing the wavelength spacing versus pulse signal with modulation in laser pumping, which is significant exponentially decreasing.

\section{Conclusion}

We have experimentally demonstrated an investigation of the multi wavelength generated in the EDFA by modulation of the laser pumping scheme. The output power of the EDFA is about $3.16 \mu \mathrm{W}$ and the spectral width is about $1.49 \mathrm{~nm}$ to $14.2 \mathrm{~nm}$. A simple analysis of the proposed system has also been reviewed. To optimize the experiment, the prototype parameters such as the optical spectral width, pulse frequency for modulation and optical power, the experimental procedures suffering from the environment are also taken into consideration. We can use this technique in the future for investigating all optical band pass active filter by EDFA.

\section{Acknowledgements}

The authors wish to thank W. Loedhammacakra, at the Department of Electronics and Telecommunications, Faculty of Engineering, Rajamangala University of Technology Thanyaburi for kindly supporting our experimental setup. We acknowledge to E. B. Manoukian for making correction of words. S. Sukhasena was supported by DRA, Naresuan University (Grant No. R2557C048).

\section{References}

[1] Yang, C., Xia, L., Wang, Y. and Liu, D. (2013) Optics \& Laser Technology, 51, 62-66.

[2] Wang, J. and Wang, H. (2014) Infrared Physics \& Technology, 65, 1-4. http://dx.doi.org/10.1016/j.infrared.2014.03.002

[3] Yeh, C.H., Chow, C.W., Chen, J.H., Chen, K.H. and Lu, S.S. (2013) Optical Fiber Technology, 19, 359-361. http://dx.doi.org/10.1016/j.yofte.2013.04.006

[4] Al-Mansoori, M.H., Adikan, F.R.M., Naji, A.W., Abdullah, M.K. and Mahdi, M.A. (2006) Optik, 117, $128-130$. http://dx.doi.org/10.1016/j.ijleo.2005.08.005

[5] Yeh, C.H., Shih, F.Y., Wen, S., Chow, C.W. and Chi, S. (2009) Optics Communications, 282, 546-549. http://dx.doi.org/10.1016/j.optcom.2008.10.038

[6] Yeh, C.H., Chow, C.W., Wu, Y.F., Shih, F.Y., Wang, C.H. and Chi, S. (2009) Optical Fiber Technology, 15, $344-347$. http://dx.doi.org/10.1016/j.yofte.2009.03.002 
[7] Yao, J., Yao, J.P., Wang, Y., Tjin, S.C., Zhou, Y., Lam, Y.L., Liu, J. and Lu, C. (2001) Optics Communications, 191, 341-345. http://dx.doi.org/10.1016/S0030-4018(01)01154-3

[8] Sheu, F.-W., Chiou, C.-Y. and Yang, S.-C. (2008) Optics Communications, 281, 4719-4722. http://dx.doi.org/10.1016/j.optcom.2008.05.038

[9] Liu, T., Wang, Q. and Soh, Y.C. (2003) Optics Communications, 225, 89-94. http://dx.doi.org/10.1016/j.optcom.2003.07.034

[10] Liu, Y.-G., Wang, D. and Dong, X. (2008) Optics Communications, 281, 5400-5404. http://dx.doi.org/10.1016/j.optcom.2008.07.081

[11] Han, Y.-G., Kim, G., Lee, J.H. and Lee, S.B. (2005) Optics Communications, 256, 98-102. http://dx.doi.org/10.1016/j.optcom.2005.06.053

[12] Sun, J., Li, H., Cheng, Y. and Li, J. (2008) Optics Communications, 281, 5874-5883. http://dx.doi.org/10.1016/j.optcom.2008.08.013 